\title{
Ossiculoplasty using Autologous Reshaped Incus and Teflon PORP: A Comparative Study
}

Ankit Choudhary, ${ }^{1}$ Sayan Hazra, ${ }^{1}$ Arindam Das, ${ }^{1}$ Aryabrata Dubey, ${ }^{1}$ Mridul Janweja, ${ }^{1}$ Arunabha Sengupta ${ }^{1}$

\section{Introduction:}

\section{ABSTRACT}

Autologous reshaped Incus and Teflon partial ossicular replacement prosthesis (PORP) are commonly used for ossicular chain reconstruction. The present study attempts to assess the post-operative outcome with these two prostheses.

Materials and Methods:

A Randomized prospective study was conducted in Tertiary referral care hospital to determine which material, among autologous reshaped Incus and Teflon partial ossicular replacement prosthesis (PORP) gives better postoperative hearing result in Ossiculoplasty.

Patients presenting at outpatients' department with the clinical diagnosis of chronic otitis media with perforation or retraction. The study population consisted of a total of 50 patients. Ossiculoplasty with reshaped Incus or PORP was performed after Canal Wall Up mastoidectomy. Hearing results were measured by Air-Bone gap in PTA after 6 months of surgery.

\section{Results:}

Selecting the criteria $<20 \mathrm{~dB} A B G$ as success when Stapes superstructure is present, Incus has $64.29 \%$ success rate, whereas PORP has $31.81 \%$ success. Extrusion rate of different prosthesis shows PORP has $18.18 \%$ extrusion whereas autologous Incus has lower (7.14\%) extrusion rate.

\section{Conclusion:}

Among these two ossiculoplasty materials, autologous Incus gives better postoperative hearing gain and lower extrusion rate. Kevwords:

Ossicular Prosthesis; Incus; Polytetrafluoroethylene; Hearing

$\mathrm{T}$ The earliest recorded attempt to reconstruct hearing in cases of chronic otitis media (COM) with ossicular discontinuity was in 1901. Since then plenty of materials have been used for ossicular substitution or reconstruction including both biological and alloplastic materials. The ideal prosthesis for ossicular reconstruction should be biocompatible, stable, safe, and easily insertable with capability of yielding optimal sound transmission. Biological materials include autograft or homograft ossicles, cortical bone and cartilage. We had undertaken a prospective study to compare autologous bone (Incus) and Teflon PORP for

1 - Department of ENT, IPGMER, Kolkata

\section{Corresponding author:}

Dr Sayan Hazra

email: sayanhazra@yahoo.co.in for reconstruction of the hearing mechanism.

\section{Materials and Methods}

The study was conducted in a tertiary care hospital of West Bengal between February 2017 and March 2018. Patients presenting at outpatients department with the clinical diagnosis of chronic otitis media with perforation or retraction were selected. A total of 69 patients were selected for this study initially. The allocation of prosthesis was determined by random number generator via software (www.graphpad.com/quickcalcs). Among 69 patients, 35 were allocated to reshaped Incus and 34 allocated to PORP. Out of which 13 patients did not meet selection criteria ( 5 from Incus group and 8 from PORP group). Hence, total 50 patients were taken in the study group, among them 23 patients were male and 27 were female. Exclusion criteria of the study were patients 


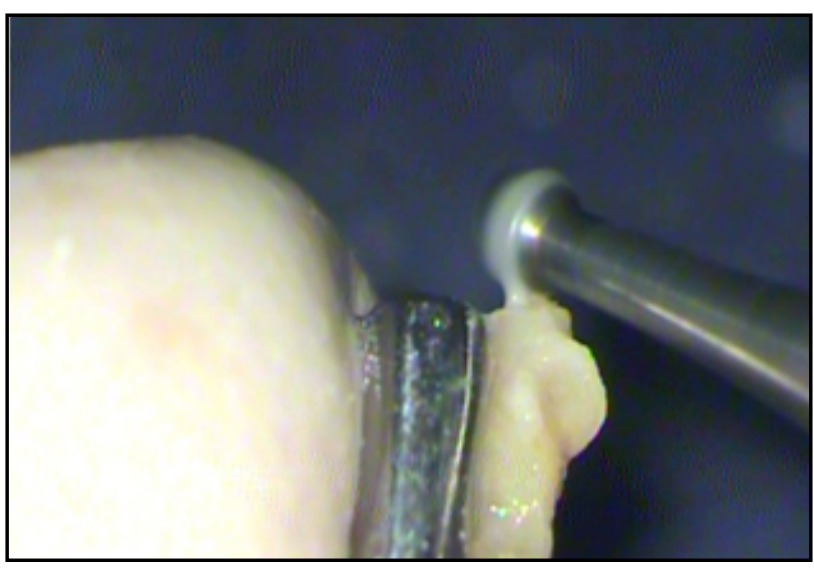

Fig.1. Homologous Incus reshaping

having cholesteatoma or any complications of COM like mastoiditis, meningitis, subdural abscess, lateral sinus thrombophlebitis, facial nerve palsy and those patients who had lost stapes superstructure or intact ossicular chain. Thirteen such patients were excluded from our study. After making the provisional diagnosis, patients underwent pre-operative haematological and radiological examinations. Pre-operative hearing status was assessed by serial tuning fork test and pure tone audiometry (PTA). The operation performed was Canal Wall Up mastoidectomy. All the surgeries were performed by same surgeon and assistant.

During the operation, it was found that most of the patients had lost only the long process of Incus but Malleus and Stapes were present. Some patients

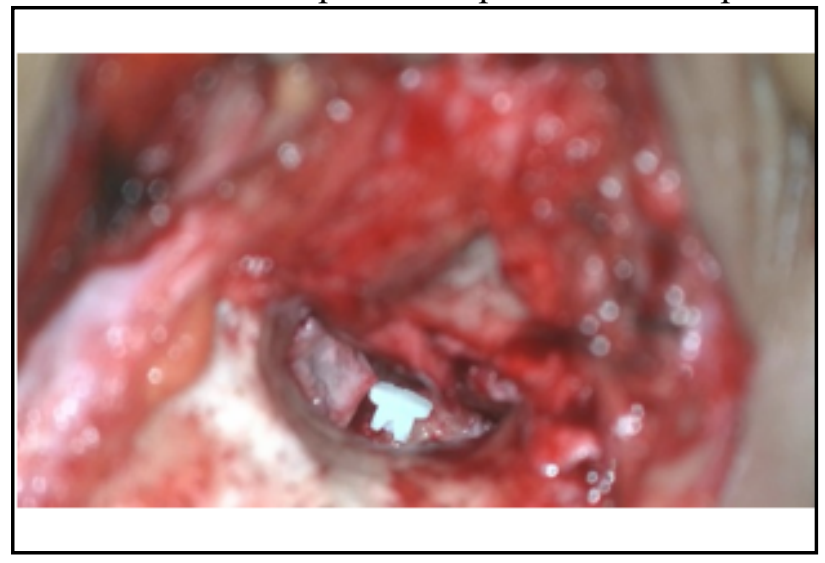

Fig. 3. Slit on PORP for engaging Stapedius tendon

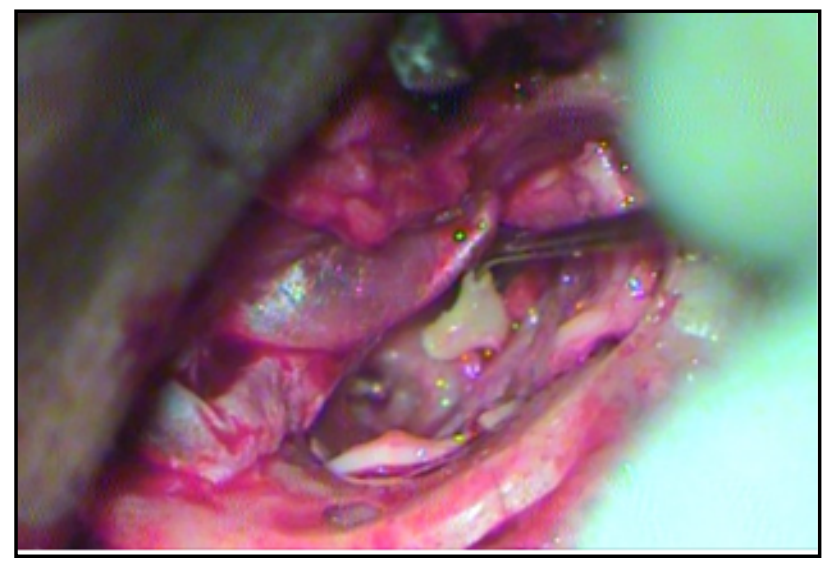

Fig. 2. Placing reshaped incus over Stapes

had lost part or whole of Malleus and/or Incus but Stapes superstructure was present. In most cases, we had remnant part or whole of the Malleus where it was kept for maintaining the integrity of remnant tympanic membrane. In all patients of COM with central perforation cortical mastoidectomy was done and ossicular integrity was checked. Patients having COM with retraction, limited atticotomy via insideout approach mastoidectomy was carried out and the retraction pocket followed till its distal end and excised. Attic defect was reconstructed with conchal cartilage. Ossicular integrity was established with reshaped Incus or Teflon PORP. Patients requiring attico-antrostomy and/or modified radical mastoidectomy for retraction pockets extending into the antrum, medial to the ossicles,

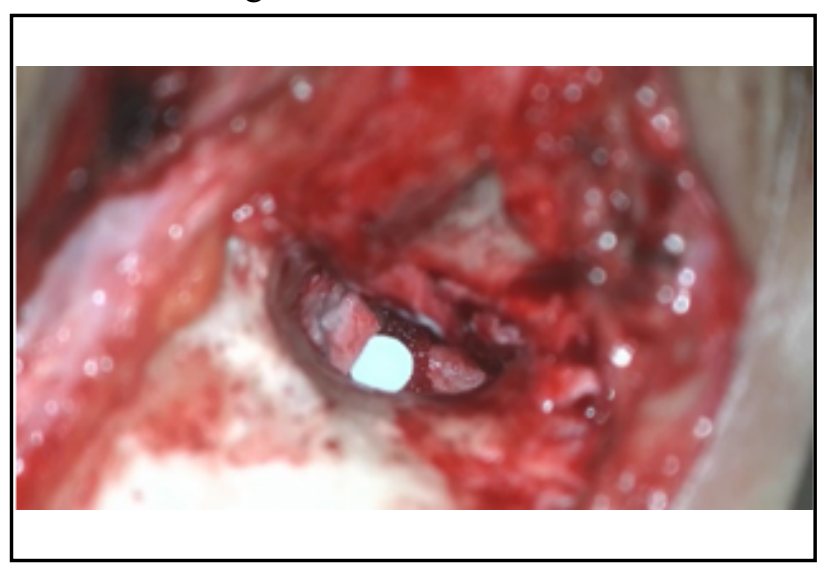

Fig. 4. PORP placed over Stapes 


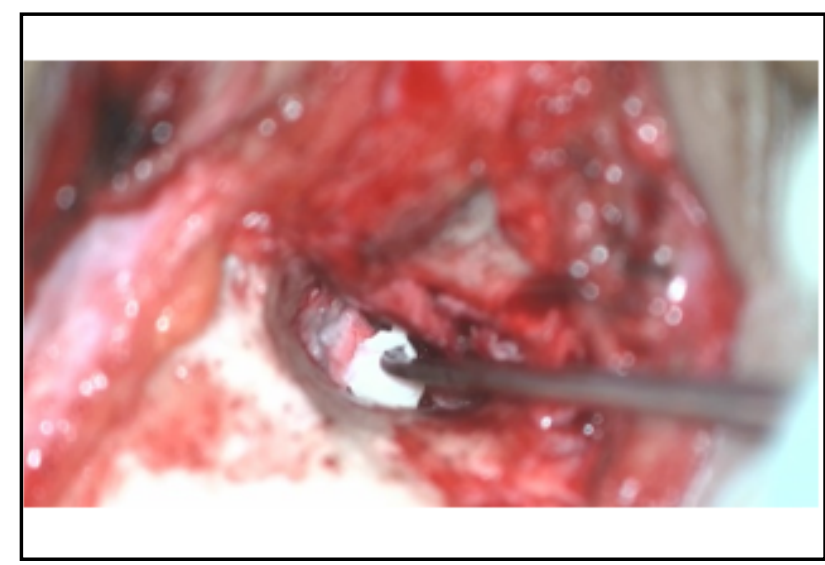

Fig. 5. Cartilage over PORP to prevent extrusion

anterior epitympanum, Eustachian tube opening were excluded from the study. Incus with necrosed lenticular/ long process was detached from the Incudo-Malleolar joint and taken out. It was then held with ossicle holding forceps such that the body of the incus was available for drilling and reshaping. Drilling was performed using 0.6 $\mathrm{mm}$ diamond burr with scanty irrigation.(Fig.1)

The remnant long process was drilled to make it cylindrical in shape with a flat base. A socket was made drilling in the under surface of reshaped long process for engaging the head of Stapes. Refashioned incus was interposed between handle of Malleus and Stapes superstructure. (Fig. 2) Then grafting was done with temporalis fascia.

In case of PORP, adequate size of the prosthesis was determined and the shaft cut accordingly. A small slit was made on the shaft of the prosthesis for engagement

Table I: Age distribution of patients

\begin{tabular}{|c|c|c|}
\hline $\begin{array}{c}\text { AGE (IN } \\
\text { YEARS) }\end{array}$ & $\begin{array}{c}\text { NUMBER OF } \\
\text { PATIENTS }\end{array}$ & $\begin{array}{c}\text { PERCENTAGE } \\
(\%)\end{array}$ \\
\hline $11-20$ & 2 & 4 \\
\hline $21-30$ & 8 & 16 \\
\hline $31-40$ & 16 & 32 \\
\hline $41-50$ & 18 & 36 \\
\hline $51-60$ & 6 & 12 \\
\hline$>60$ & 0 & 0 \\
\hline
\end{tabular}

of Stapedius muscle for better stability of the prosthesis. (Figs. 3\&4) A small piece of cartilage was placed between prosthesis head and temporalis fascia graft to prevent direct contact and thus reduce the extrusion. (Fig.5) During surgery, type of prosthesis was selected randomly. After the operation, patients were discharged on 3rd post-operative day and routinely followed-up at 1-week, 4 weeks, 3 months and 6 months.

\section{Results}

Among the 50 patients of our study, 28 underwent autologous reshaped Incus ossiculoplasty and 22 underwent ossiculoplasty using PORP. The outcome was studied at the end of 6 months. All the patients were above 12 years of age. (Table I)

Among 50 patients, 22 patients had granulation tissue, 17 patients had oedematous mucosa as pathology in the middle ear cleft and 11 patients had retraction pocket. Preoperative hearing status was recorded as follows. (Table II)

From the above chart, it is seen that when reshaped Incus is used as prosthetic material, $35.66 \%$ patients have AB Gap $>20 \mathrm{~dB}$. Whereas Teflon PORP has $>20$ $\mathrm{dB}$ AB Gap in $68.17 \%$ cases.

Post-operative $\mathrm{AB}$ Gap of $<20 \mathrm{~dB}$ was considered to be the yardstick for successful ossiculoplasty. (Table IV) It was observed that ossiculoplasty using reshaped Incus had a success of $64.29 \%$ whereas Teflon PORP has success of $31.81 \%$.

Extrusion rate in patients who underwent

Table II: Preoperative hearing status. The above chart shows that most of the patients had preoperative $A B G$ in between 40 and $50 \mathrm{~dB}$.

\begin{tabular}{|c|c|}
\hline $\begin{array}{c}\text { PRE-OPERATIVE AB } \\
\text { GAP (DB) }\end{array}$ & $\begin{array}{c}\text { NUMBER OF } \\
\text { PATIENTS (\%) }\end{array}$ \\
\hline $30-40$ & $18(36)$ \\
\hline $40-50$ & $21(42)$ \\
\hline $50-60$ & $8(16)$ \\
\hline$>60$ & $3(6)$ \\
\hline
\end{tabular}


Table III: The postoperative hearing status with different graft materials

\begin{tabular}{|c|c|c|}
\hline POST-OPERATIVE AB GAP (DB) & $\begin{array}{c}\text { RESHAPED AUTOLOGOUS } \\
\text { INCUS (N=28) (\%) }\end{array}$ & TEFLON PORP (N=22) (\%) \\
\hline $0-10$ & $03(10.71)$ & 0 \\
\hline $11-20$ & $15(53.57)$ & $07(31.81)$ \\
\hline $21-30$ & $06(21.43)$ & $07(31.81)$ \\
\hline$>30$ & $04(14.23)$ & $08(36.36)$ \\
\hline
\end{tabular}

Table IV: Post-Operative Air-Bone Gap $<20 \mathrm{~dB}$ as a yardstick of successful ossiculoplasty.

Chi-square statistic is 5.1948 with a p-value of 0.022654 which is significant at $p<0.05$.

\begin{tabular}{|c|c|c|c|}
\hline $\begin{array}{c}\text { PROSTHETIC } \\
\text { MATERIAL }\end{array}$ & $\begin{array}{c}\text { SUCCESS (POST-OP } \\
\text { ABG }<20 \text { DB) }\end{array}$ & $\begin{array}{c}\text { FAILURE (POST-OP } \\
\text { ABG }>20 \text { DB) }\end{array}$ & TOTAL CASES \\
\hline Reshaped Incus & $64.29(18)$ & $35.71(10)$ & 28 \\
\hline PORP & $31.81(07)$ & $68.18(15)$ & 22 \\
\hline
\end{tabular}

Table V: Extrusion rate in patients who underwent ossiculoplasty with different materials.

Chi-square statistic is $\mathbf{1 . 4 2 1 7}$ with a p-value of 0.233126 which is not significant at $\mathbf{p}<\mathbf{0 . 0 5}$.

\begin{tabular}{|c|c|c|l|}
\hline PROSTHETIC MATERIAL & EXTRUSION & $\begin{array}{c}\text { NUMBER OF } \\
\text { PATIENTS }\end{array}$ & $\begin{array}{l}\text { P E R C E N T A G E } \\
(\%)\end{array}$ \\
\hline Reshaped Incus & 2 & 28 & 7.14 \\
\hline PORP & 4 & 22 & 18.18 \\
\hline
\end{tabular}

ossiculoplasty with different materials $(\mathrm{n}=50)$ is shown in Table V. It was observed that reshaped Incus had an extrusion in only $7.14 \%$ of cases, whereas Teflon PORP had an extrusion rate of $18.18 \%$.

\section{Discussion}

Various materials and surgical techniques have been employed for ossicular chain reconstruction since the early 20th Century but still there is no ideal material and/or standardized technique that has been accepted worldwide. Otologists around the globe use three general classes of prosthesis now-a-days viz; autograft, homograft and allograft.

Autograft prosthesis include ossicles (incus, malleus), cartilage (septal spur, tragal), cortical bone. Advantages of autograft include biocompatibility, very low extrusion rate and no risk of transmitting disease. Displacement, partial or complete absorption and possibility of harbouring microscopic disease have been blamed as potential disadvantages of their use. ${ }^{1}$

Homografts are collected from cadavers or healthy person, stored in alcohol and then used for ossiculoplasty. Main disadvantages of these grafts are transmission of prion diseases. For this reason and as a result of ready availability of ossicular prosthesis, this practice has been minimized. ${ }^{2,3}$

Allograft prosthesis is readily available, presculptured and made of various synthetic materials such as Teflon, hydroxyapatite, plastipore, titanium, glass-ceramics, etc., and are designed to be made biocompatible. Ossicular necrosis, extrusion, displacement and unsatisfactory hearing outcome have been encountered with virtually every type of design. Extrusion of 
prosthesis has been reported as high as $39 \%$. However, it can be significantly reduced by placing cartilage or bone between the remnant tympanic membrane or graft and the prosthesis being used. ${ }^{4}$

Success in ossiculoplasty is determined by technical ability and to a large extent case selection. Much of the variability in the literature concerning hearing results after ossiculoplasty is due to lack of understanding and uniform reporting of those middle ear factors that influence the results. ${ }^{5,6}$ It was observed that the most common type of ossicular chain erosion encountered is necrosis of the long process of the Incus because of its anatomical position and course of its blood supply. ${ }^{7,8}$ When the Stapes superstructure is missing, the aim is to achieve a postoperative air-bone gap $(\mathrm{ABG})<30 \mathrm{~dB}$, when Stapes is intact, an $\mathrm{ABG}<20 \mathrm{~dB}$ is acceptable. While using these criteria, the Incus interposition appears to be more successful than the use of the prosthesis.

Jha et al in 2007-2009 performed a study of ossiculoplasty outcome after 2 and 5 months of operation in relation to $\mathrm{ABG}$ and suggested success rate with cartilage was $57 \%$, with incus it was $59 \%$ and for plastic PORP and total ossicular replacement prosthesis (TORP) it was (40\%). ${ }^{9}$ Another study on outcome of ossiculoplasty showed success rate of incus was $58 \%$, where as in the case of PORP and TORP was $33 \%{ }^{10}$ Gardner et al. published a retrospective study comparing the success results of PORP and TORP showing 48\% in case of PORP and $24 \%$ in case of TORP when polyethylene-based prostheses were used. ${ }^{11}$

In our study, 50 cases were included out of initial selection of 69 cases. Among those, 28 patients underwent autologous incus ossiculoplasty, whereas 22 reconstructions were with Teflon PORP. The success rate was $64.29 \%$ in case of Incus, $31.81 \%$ in case of PORP after 6 months of postoperative follow-up ( $A B$ Gap $<20 \mathrm{~dB}$ when Stapes superstructures is present, is considered as success).

Jha et al, in their comparative study on ossiculoplasty, described that the failure and extrusion in case of cartilage were $11.5 \%$, in bone $5.9 \%$ and in plastic PORP it was 20\%.9 Our study too, showed higher extrusion rate in case of prosthesis. Among 28 cases autologous Incus ossiculoplasty, 2 cases of extrusion occurred (7.14\%).
Out of 22 Teflon PORP cases, 4 cases of extrusion were observed $(5.26 \%)$.

The mass and stiffness of prostheses may influence sound conduction in a frequency dependent manner. Studies by Meister et al. ${ }^{12}$ and Kelly et al. ${ }^{13}$ have suggested mass of the prosthesis as the most important variable and prostheses weight should be as light as possible to optimize transmission of frequencies above $1000 \mathrm{~Hz}$. More recent mathematical model by Zenner et al. ${ }^{14}$ has determined that a mass of $5 \mathrm{mg}$ or less provides maximum transfer of energy. The coupling of a prostheses to the tympanic membrane (or manubrium) and the stapes superstructure to footplate also influence overall result. The probable cause of incus giving the best postoperative result may be due to low impedance of incus compared to cartilage and PORP. Another reason may be the incudo-stapedeal joint movement which is best with the Incus compared to cartilage or PORP. When Incus is used as prosthesis, rocking movement of stapes footplate is maintained but in case of PORP, it is a transitional movement leading to less hearing gain compared to Incus prosthesis. From an economic point of view, a good quality of Plastipore prosthesis costs around 5000 rupees and for Titanium prosthesis, it is more than 10,000 rupees. COM mainly affects poor socioeconomic patients and for them bearing an additional cost of the prosthesis become very difficult. Our study was done in a Government tertiary care centre where all the surgeries were performed free of cost.

\section{Conclusion}

Comparative study on hearing outcome of ossiculoplasty and extrusion rates using different materials in different ossicular status of middle ear had already been done by various authors and still many more researches are ongoing across the globe. Though different prosthesis materials produce different vibrational response, a biocompatible and mechanically stable implant produces improvement in hearing of patients with less extrusion rate.

In this study, we have shown that hearing results after ossiculoplasty with reshaped autologous incus is significantly better as compared to those obtained with 
Teflon PORP. Furthermore, autologous incus has very low extrusion rate compared to other PORP.

With a small sample size in this study, there is chance of statistical error. To combat this shortcoming, prospective clinical studies with larger sample size are required to assess the long-term outcome of ossiculoplasty, but compliance for longer follow-up is a matter of concern. It is worthwhile to conclude that there is a need for many clinical trials with larger sample size and longer followup period to standardize the ossiculoplasty techniques and accept an ideal ossicular prosthesis which will stand the test of time.

With the continuing advances in our understanding of middle ear mechanics, the outcome of ossiculoplasty is improving. Careful attention to the principles of ossicular reconstruction and applying them in clinical practice makes it possible to give more desirable hearing results for the patients.

\section{References}

1. Kartush JM. Ossicular chain reconstruction, Capitulum to malleus, Otolaryngol Clin North Am. 1994; 27:689-715

2. Lubbe D, Fagan JJ. Revisiting the risks involved in using homograft ossicles in otological surgery. $\mathrm{J}$ Laryngol Otol. 2008;122:111-5

3. Glasscock ME 3 rd, Jackson CG, Knox GW. Can acquired immunodeficiency syndrome and Creutzfeldt-Jakob disease be transmitted via otologic homografts? Arch Otolaryngol Head Neck Surg. 1988;114:1252-5
4. Toner JC, Smyth GD, Kerr AG. Realities in ossiculoplasty. J Laryngol Otol. 1991; 105:529-33

5. Black B. Ossiculoplasty prognosis: The spite method of assessment. Am J Otol. 1992;13:544-51

6. Albu S, Babighian G, Trabalzini F. Prognostic factors in tympanoplasty. Am J Otol. 1998;19:136-40

7. O'Reilly RC, Cass SP, Hirsch BE, Kamerer DB, Bernat RA, Poznanovic SP. Ossiculoplasty using incus interposition: Hearing results and analysis of the middle ear risk index. Otol Neurotol. $2005 ; 26: 853-8$

8. Athanasiadis-Sismanis A, Poe DS. Ossicular chain reconstruction. In: Gulya AJ, Minor LB, Poe DS Eds, GlasscockShambaugh Surgary of the Ear, 6 th ed. Ch. 29. USA: People's Medical Publishing House, 2010: p489-500

9. Jha S, Mehta K, Prajapati V, Patel D, Kharadi P. A comparative study of ossiculoplasty by using various graft materials. NJIRM $2011 ; 2: 53-7$

10. Amith I, Naragund RS Mudhol, Harugop AS, Patil PH. Ossiculoplasty with autologous incus vs prosthesis: A comparison of anatomical and functional results. Indian Journal of Otology $2011 ; 17: 75-9$

11. Gardner EK, Jackson CG, Kaylie DM. Results with titanium ossicular reconstruction prostheses. Laryngoscope 2004;114:65-70

12. Meister H, Walger M, Mickenhagen A, von Wedel H, Stennert E. Standardized measurements of the sound transmission of middle ear implants using a mechanical middle ear model. Eur Arch Otorhinolaryngol. 1999; 256:122-7

13. Kelly DJ, Prendergast PJ, Blayney AW. The effect of prosthesis design on vibration of the reconstructed ossicular chain: A comparative finite element analysis of four prostheses, Otol Neurotol. 2003; 24:11-9

14. Zenner HP, Stegmaier A, Lehner R, Baumann I, Zimmermann R. Open Tübingen titanium prostheses for ossiculoplasty: A prospective clinical trial. Otol Neurotol. 2001; 22:582-9. 